\title{
A SIMPLIFIED EXPRESSION OF SHARE FUNCTIONS FOR COOPERATIVE GAMES WITH FUZZY COALITIONS
}

\author{
Rajib Biswakarma - Surajit Borkotokey - RAdko Mesiar
}

\begin{abstract}
In this paper, we discuss the notion of Share functions for cooperative games with fuzzy coalitions or simply fuzzy cooperative games. We obtain the Share functions for some special classes of fuzzy games, namely the fuzzy games in proportional value form and the fuzzy games in Choquet integral form. The Shapley Share and Banzhaf Share functions for these classes are derived.
\end{abstract}

\section{Introduction}

A cooperative game with transferable utility, or simply a TU-game, is a pair $(N, v)$, where $N$ is a finite set of players and $v$, a characteristic function defined on $2^{N}$ that assigns every subset (coalition) a real number called its worth giving zero worth to the empty coalition. It is assumed that the players form binding agreements and the worth is generated under cooperation by virtue of this binding agreement. A solution to a cooperative game is an $n$-dimensional vector of real numbers that assigns each player her payoff. A value function for TU-games is a function that assigns a solution to every cooperative game. A value function is efficient if the payoffs to the players add up to the worth of the grand coalition. The Shapley value [12] and the B a $\mathrm{zh}$ af value [6] are perhaps the most famous efficient and inefficient values, respectively. Share functions for TU-games due to van der Laan and van den Brink [16] are

(C) 2017 Mathematical Institute, Slovak Academy of Sciences.

2010 Mathematics Subject Classification: 91A12, $62 \mathrm{C} 86$.

Keywords: fuzzy cooperative game, Shapley function, Banzhaf function, Share function, Shapley Share function, Banzhaf Share function, Choquet integral.

This work is partially supported by the grant VEGA 1/0420/15 and MRP, UGC-India 42-26/2013(SR). The second author acknowledges the support of SAIA, Slovakia and the Department of Mathematics, Slovak University of Technology during his visit there for two months. 


\section{RAJIB BISWAKARMA — SURAJIT BORKOTOKEY — RADKO MESIAR}

a type of solutions that assign to every game a vector whose components add up to one. A Share function determines how much share a player can get from the worth of the grand coalition and therefore is devoid of the efficiency requirement as opposed to the other standard value functions. Therefore a Share function simplifies the model formulation to a great extent. The Share function corresponding to the Shapley value (Banzhaf value) is the Shapley Share function (Banzhaf Share function). It is obtained by dividing the Shapley value (Banzhaf value) of each player by the sum of the Shapley values (Banzhaf values) of all players. Cooperative games with fuzzy coalitions or simply fuzzy games are generalization of ordinary TU-games in the sense that participation of the players is considered here to be partial that ranges between 0 and 1, see [1. A fuzzy coalition is a fuzzy subset of the player set $N$ that assigns a membership grade to its members that represents player's rate of participation in it.

In this paper, we propose the notion of Share functions for fuzzy games. A set of axioms to characterize the Share function is proposed. Some interesting results pertaining to a special class of fuzzy games, namely the fuzzy games in Choquet integral and proportional value form are obtained. The Shapley Share function for this class is derived as an illustration of the model.

The rest of the paper proceeds as follows. In Section 2, we compile the related definitions and results from the existing literature. Section 3 discusses the Share functions for fuzzy cooperative games. In Section 4, we discuss the Share functions for games in proportional value form followed by the Share functions for games in Choquet integral form along with their corresponding examples. Section 5 concludes.

\section{Preliminaries}

In this section, we compile the related definitions and results from [9, 14]-17] relevant to the development of the paper for ready reference. Let the player set $N$ be fixed. A TU-game $(N, v)$ comprises of the player set $N$ and the characteristic function $v: 2^{N} \rightarrow \mathbb{R}$ such that $v(\emptyset)=0$. If there is no ambiguity on $N$, we denote the TU-game $(N, v)$ by its characteristic function $v$ only. Let $G_{0}(N)$ denote the class of all crisp TU-games with player set $N$. The game $v \in G_{0}(N)$ is superadditive if for each $S, T \subseteq N, S \cap T=\emptyset, v(S \cup T) \geq v(S)+v(T)$. Players $i, j \in N$ satisfying: $v(K \cup i)=(K \cup j)$ for every $K \subseteq N \backslash\{i, j\}$ are called symmetric players. The axiomatization of the Share function makes use of the notion of a carrier of a coalition which is defined as follows.

Definition 1. Let $v \in G_{0}(N)$ and $K \in 2^{N}$. A coalition $S \subseteq K$ is called a carrier in $K$ for $v$ if $v(S \cap T)=v(T), \forall T \in 2^{K}$. 


\section{A SIMPLIFIED EXPRESSION OF SHARE FUNCTIONS FOR COOPERATIVE GAMES}

The set of all carriers in $K$ for $v$ denoted by $C\left(\left.K\right|_{v}\right)$ is therefore given by

$$
C\left(\left.K\right|_{v}\right)=\{S \subseteq K \mid v(S \cap T)=v(T), \quad \forall T \subseteq K\} .
$$

Similarly, the null player is defined as follow.

Definition 2. Given $v \in G_{0}(N)$. Player $i$ is called a null player of $v$ if for any $T \subseteq N \backslash i, v(T \cup i)=v(T)$.

For any $S \subseteq N$, we denote by $N(S)$, the set of players $i \in N \backslash S$ such that $v(T \cup i)=v(T)$ whence $T \subseteq S$. Thus for any $T \subseteq N(S)$, we have $v(T)=v(S \cup T)$.

A solution of an $n$-person TU-game is an $n$-dimensional vector representing a distribution of payoffs. A value function on a subset $C$ of $G_{0}(N)$ is a function that assigns a solution to any game in $C$. A value function $\Phi$ is efficient on $C$ if $\sum_{i=1}^{n} \Phi_{i}(N, v)=v(N)$ for all $(N, v) \in C$.

The Shapley value $\Phi_{i}^{S h}$ of player $i$ with respect to a game $v \in G_{0}(N)$ and for all $K \subseteq N$ is a weighted average value of the marginal contributions $v(T)-v(T \backslash i)$ of player $i$ in all combinations. It is given by

$$
\Phi_{i}^{S h}(K, v)=\sum_{\left\{T: i \in T \subset K \in 2^{N}\right\}} \frac{(|T|-1) !(|K|-1) !}{|K| !}[v(T)-v(T \backslash i)] .
$$

The Banzhaf value $\Phi_{i}^{B}$ of player $i$ with respect to a game $v \in G_{0}(N)$ and for all coalitions $K \subseteq N$ is a weighted average value of the marginal contributions $v(T)-v(T \backslash i)$ of player $i$ alone in all combinations, which is given by

$$
\Phi_{i}^{B}(K, v)=\sum_{\left\{T: i \in T \subset K \in 2^{N}\right\}} \frac{1}{2^{|K|-1}}[v(T)-v(T \backslash i)] .
$$

In what follows next we define the Share functions for a class of crisp games followed by the definitions of the corresponding Shapley and Banzhaf Share functions.

\subsection{Share functions on the class of crisp games}

Let $\mu: G_{0}(N) \rightarrow \mathbb{R}$ be a function assigning a real value to any game $v \in G_{0}(N)$. Then $\mu$ is positive on $C \subseteq G_{0}(N)$ if $\mu(v)>0$ for all $v \in C$, and zero on $C \subseteq G_{0}(N)$ if $\mu(v)=0$ for all $v \in C$. We call $\mu$ additive on $C \subseteq G_{0}(N)$ if for every pair of games $v, w \in C$ such that $v+w \in C$ it holds that $\mu(v+w)=\mu(v)+\mu(w)$. $\mu$ is linear on $C$ if it is additive on $C$ and for every $v \in C$ and $\alpha \in \mathbb{R}$ such that $\alpha v \in C$ it holds that $\mu(\alpha v)=\alpha \mu(v)$. Finally, we call $\mu$ symmetric on $C$ if for every $v \in C$, every pair of symmetric players $i, j \in N$ and every $E \subset N$; $E \supset\{i, j\}$, such that the subgames $\left(E \backslash\{i\}, v_{E \backslash\{i\}}\right)$ and $\left(E \backslash\{j\}, v_{E \backslash\{j\}}\right)$ are in $C$, it holds that $\mu\left(E \backslash\{i\}, v_{E \backslash\{i\}}\right)=\mu\left(E \backslash\{j\}, v_{E \backslash\{j\}}\right)$.

In the following, we introduce the Share function with respect to a function $\mu: C \rightarrow \mathbb{R}$ on a class $C$ of crisp games along the line of $[15]$ which is both specific 


\section{RAJIB BISWAKARMA — SURAJIT BORKOTOKEY — RADKO MESIAR}

to the game and the coalition. Note that a Share function assigns to each player his share in the payoff $v(K)$ of the coalition $K \subseteq N$, i.e., a Share function on a class $C \subseteq G_{0}(N)$ of games is a function that gives player $i$ her share in the value $v(K)$ of the coalition $K \subseteq N$. The formal definition goes as follows.

Definition 3. Let $\mu: C \rightarrow \mathbb{R}$ be given. A Share function on a set of games $C \subseteq G_{0}(N)$ is a function $\Psi^{\mu}$ that assigns to every game $(N, v) \in C$ an $n$-dimensional real vector $\Psi^{\mu}(N, v)$ such that the following axioms hold.

Axiom $\mathbf{S}_{1}$ : If $v \in C$ and $K \in 2^{N}$ and $T \in C\left(\left.K\right|_{v}\right)$ then,

$$
\sum_{i \in T} \Psi_{i}^{\mu}(K, v)=1
$$

and

$$
\Psi_{i}^{\mu}(K, v)=0, \quad i \notin T
$$

Axiom $\mathbf{S}_{2}$ : If $v \in C$ and $K \in 2^{N}, i, j \in K$ and $v(S \cup\{i\})=v(S \cup\{j\})$ holds for any $S \subseteq K \backslash\{i, j\}$ then $\Psi_{i}^{\mu}(K, v)=\Psi_{j}^{\mu}(K, v)$.

Axiom $\mathbf{S}_{\mathbf{3}}$ ( $\mu$-additivity): For any pair $v, w \in C$ such that $v+w \in C$, it holds that $\mu(K, v+w) \Psi_{i}^{\mu}(K, v+w)=\mu(K, v) \Psi_{i}^{\mu}(K, v)+\mu(K, w) \Psi_{i}^{\mu}(K, w)$.

The following theorem is due to [16].

Theorem 1. Let $\mu: C \rightarrow \mathbb{R}$ be a positive function on $C$. Then on the subclass $C$ there exists a unique Share function $\Psi^{\mu}: C \rightarrow\left(\mathbb{R}_{+} \cup\{0\}\right)^{2^{N}}$ satisfying the axioms $\mathbf{S}_{\mathbf{1}}-\mathbf{S}_{\mathbf{3}}$ if and only if $\mu$ is additive on $C$.

Note that a Share function is always dependent on the given additive function $\mu: C \rightarrow \mathbb{R}$ and therefore unless $\mu$ has a specific form as can be seen in the following theorems, we call it a $\mu$-Share function. In the following we state the existence theorems of the Shapley and the Banzhaf Share functions for crisp games without proof.

Theorem 2. Let $C \subseteq G_{0}(N)$ and $\mu_{S}: C \rightarrow \mathbb{R}$ be defined by $\mu_{S}(K, v)=v(K)$. Then the Shapley Share function $\Psi^{\mu_{S}}(K, v)=\frac{\Phi_{i}^{S h}(K, v)}{v(K)}$ is the unique $\mu_{S}$-Share function satisfying the Axioms $S_{1}-S_{3}$ on the class $C$.

Theorem 3. Let $C \subseteq G_{0}(N)$ and $\mu_{B}: C \rightarrow \mathbb{R}$ be defined by $\mu_{B}(K, v)=$ $\sum_{S \subset K}(2|S|-|K|) v(S)$. Then the Banzhaf Share function $\Psi^{\mu_{B}}(K, v)=\frac{\Phi_{i}^{B}(K, v)}{\mu_{B}(K, v)}$ is the unique $\mu_{B}$-Share function satisfying the Axioms $S_{1}-S_{3}$ on the class $C$. 


\section{A SIMPLIFIED EXPRESSION OF SHARE FUNCTIONS FOR COOPERATIVE GAMES}

\subsection{Fuzzy cooperative games}

In this section, we make a brief description of a cooperative game with fuzzy coalitions or in short a fuzzy cooperative game. We call $\mathbf{x}=\left(x_{1}, x_{2}, \ldots, x_{n}\right)$, $x_{i} \in[0,1]$, the fuzzy coalition variable, and denote $\mathbf{x}^{f}=\left\{\mathbf{y} \mid y_{i}=x_{i}\right.$ or $y_{i}=0$ for each $i \in N\}$ the set of fuzzy coalition variables created by $\mathbf{x}$. The level set of $\mathbf{x}$ is $L(\mathbf{x})=\left\{x_{i} \mid i \in A\right\}$. The $n$-tuple $\mathbf{s}=\left(s_{1}, s_{2}, \ldots, s_{n}\right)$ is called a fuzzy coalition, here $s_{i}$ is a constant which denotes the participation level of player $i$ in the coalition $\mathbf{s}$. Thus following the same nomenclature as of $\mathbf{x}^{f}$ we have $\mathbf{s}^{f}=$ $\left\{\mathbf{t} \mid t_{i}=s_{i}\right.$ or $t_{i}=0$ for each $\left.i \in N\right\}$. Let $L(N)$ denote the set of fuzzy coalitions. The empty coalition in fuzzy setting is $\mathbf{e}^{\emptyset}=(0,0, \ldots, 0)$. A crisp coalition is given by $\mathbf{e}^{s}=\left(s_{1}, s_{2}, \ldots, s_{n}\right)$ satisfying $s_{i}=1$ when $i \in S$ and $s_{i}=0$ when $i \in N \backslash S$, with $S \in 2^{N}$. It corresponds to the situation where the players within $S$ fully cooperate, that is, they have participation level 1, and the players outside $S$ are not involved at all, i.e., they have the participation level 0. $\mathbf{e}^{N}=(1,1, \ldots, 1)$ is called the grand coalition. We denote the singleton fuzzy set $\mathbf{e}^{\{i\}}$ by $\mathbf{e}^{i}$. Let $T \subseteq N$, the fuzzy coalition variable $\mathbf{x}_{T}$ corresponding to $T$ is denoted by $\mathbf{x}_{T}=\sum_{k \in T} x_{k} \mathbf{e}^{k}$. Thus under this notation any fuzzy coalition $\mathbf{s}$ can be denoted by $\mathbf{s}=\sum_{k \in S} s_{k} \mathbf{e}^{k}$. Denote by $\operatorname{Supp}(\mathbf{s})=\left\{i \in N \mid s_{i}>0\right\}$ the support of a fuzzy coalition $\mathbf{s}$. A cooperative game with fuzzy coalitions on $N$ or a fuzzy game in short, is a function $v: L(N) \rightarrow \mathbb{R}_{+} \cup\{0\}=\{r \in \mathbb{R} \mid r \geq 0\}$ such that $v\left(\mathbf{e}^{\emptyset}\right)=0$. The class of all fuzzy games with player set $N$ is denoted by $F G(N)$.

Definition 4. A fuzzy game $v \in F G(N)$ is convex if $v(\mathbf{s} \vee \mathbf{t})+v(\mathbf{s} \wedge \mathbf{t}) \geq$ $v(\mathbf{s})+v(\mathbf{t})$ for all $\mathbf{s}, \mathbf{t} \in L(N)$, where $\mathbf{s} \vee \mathbf{t}=\left(\max \left\{s_{1}, t_{1}\right\}, \ldots, \max \left\{s_{n}, t_{n}\right\}\right)$ and $\mathbf{s} \wedge \mathbf{t}=\left(\min \left\{s_{1}, t_{1}\right\}, \ldots, \min \left\{s_{n}, t_{n}\right\}\right)$, respectively.

Definition 5. Given $\mathbf{s} \in L(N)$, let $\mathbf{s}^{p}=\left\{i \in N \mid s_{i}=p\right\}$ for any $p \in[0,1]$. A game $v \in F G(N)$ is said to be in proportional value form if it can be represented as,

$$
v(\mathbf{s})=\sum_{p \in[0,1]} v\left(\mathbf{s}^{p}\right) \cdot p \quad \text { for all } \quad \mathbf{s} \in L(N) .
$$

The set of all fuzzy games in proportional value form is denoted by $F G_{p}(N)$.

Definition 6. Given $\mathbf{s} \in L(N)$, let $Q(\mathbf{s})=\left\{s_{i} \mid s_{i}>0, i \in N\right\}$ and let $q$ (s) be the cardinality of $Q(\mathbf{s})$. We write the elements of $Q(\mathbf{s})$ in the increasing order as $h_{1}<\cdots<h_{q(\mathbf{s})}$. Then a game $v \in F G(N)$ is said to be a fuzzy game with Choquet integral form if,

$$
v(\mathbf{s})=\sum_{l=1}^{q(\mathbf{s})} v\left([\mathbf{s}]_{h_{l}}\right)\left(h_{l}-h_{l-1}\right) \quad \text { for any } \mathbf{s} \in L(N), \quad \text { where } h_{0}=0 .
$$

The set of all fuzzy games with the Choquet integral form is denoted by

$$
F G_{C}(N) \text {. }
$$




\section{RAJIB BISWAKARMA — SURAJIT BORKOTOKEY — RADKO MESIAR}

The next four theorems are of the existence and uniqueness of the Shapley and Banzhaf functions for the classes $F G_{p}(N)$ and $F G_{C}(N)$, respectively.

TheOREM $4([10])$. Let $v \in F G_{p}(N)$ and $\mathbf{s} \in L(N)$, a function $\Phi^{S h}: F G_{p}(N) \rightarrow$ $\left(\mathbb{R}_{+}^{n}\right)^{L(N)}$, defined by

$$
\Phi_{i}^{S h}(\mathbf{s}, v)= \begin{cases}\sum_{i \in T \subseteq \mathbf{s}^{p}} \frac{(|T|-1) !\left(\left|s^{p}\right|-|T|\right) !}{\left|s^{p}\right| !}\{v(T)-v(T \backslash i)\}, & \text { if } i \in \mathbf{s}^{p}, p \in(0,1] \\ 0, & \text { elsewhere }\end{cases}
$$

is the unique Shapley function in $\mathbf{s}$ for $v \in F G_{p}(N)$, where $\left|\mathbf{s}^{p}\right|$ is the cardinality of $\mathbf{s}^{p}$.

Theorem 5. Let $v \in F G_{p}(N)$ and $\mathbf{s} \in L(N)$, A function $\Phi^{B}: F G_{p}(N) \rightarrow$ $\left(\mathbb{R}_{+}^{n}\right)^{L(N)}$, defined by

$$
\Phi_{i}^{B}(\mathbf{s}, v)= \begin{cases}\sum_{i \in T \subseteq \mathbf{s}^{p}} \frac{1}{2^{\mathbf{s}^{p} \mid-1}}\{v(T)-v(T \backslash i)\}, & \text { if } i \in \mathbf{s}^{p}, p \in(0,1] \\ 0, & \text { elsewhere }\end{cases}
$$

is the unique Banzhaf function in $\mathbf{s}$ for $v \in F G_{p}(N)$.

Theorem $6([15])$. Let $v \in F G_{C}(N)$ and $\mathbf{s} \in L(N)$. A function $\Phi^{S h}: F G_{C}(N) \rightarrow$ $\left(\mathbb{R}_{+}^{n}\right)^{L(N)}$, defined by

$$
\Phi_{i}^{S h}(s, v)=\sum_{l=1}^{q(s)} \Phi_{i}^{\prime S h}\left([\mathbf{s}]_{h_{l}}, v\right) \cdot\left(h_{l}-h_{l-1}\right)
$$

is a Shapley function in $\mathbf{s}$ for $v \in F G_{C}(N)$, where

$$
\Phi_{i}^{\prime S h}\left([\mathbf{s}]_{h_{l}}, v\right)= \begin{cases}\sum_{i \in T \subseteq[\mathbf{s}]_{h_{l}}} \frac{(|T|-1) !\left(\left|[\mathbf{s}]_{h_{l}}\right|-|T|\right) !}{\left|[\mathbf{s}]_{h_{l}}\right| !}\{v(T)-v(T \backslash i)\}, & \text { if } i \in[\mathbf{s}]_{h_{l}} \\ 0, & \text { elsewhere }\end{cases}
$$

is the Shapley function of the crisp game $v$.

Theorem 7 (14]). Let $v \in F G_{C}(N)$ and $\mathbf{s} \in L(N)$. A function $\Phi^{B}: F G_{C}(N) \rightarrow$ $\left(\mathbb{R}_{+}^{n}\right)^{L(N)}$, defined by

$$
\Phi_{i}^{B}(\mathbf{s}, v)=\sum_{l=1}^{q(s)} \Phi_{i}^{\prime B}\left([s]_{h_{l}}, v\right) \cdot\left(h_{l}-h_{l-1}\right)
$$

is a Banzhaf function in $\mathbf{s}$ for $v \in F G_{C}(N)$, where

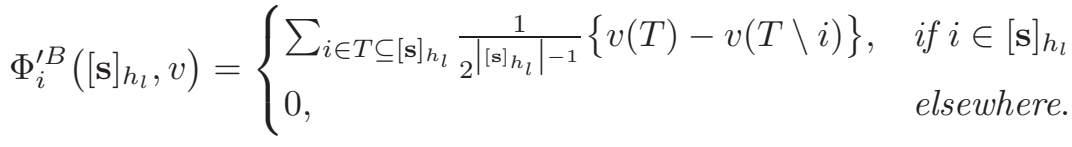




\section{A SIMPLIFIED EXPRESSION OF SHARE FUNCTIONS FOR COOPERATIVE GAMES}

\section{Share functions for fuzzy games}

We now extend the notion of a Share function to the class $F G(N)$ of fuzzy games with player set $N$. Following its crisp counterpart we assume here also that the Share function assigns to each player her share in the payoff $v(\mathbf{s})$ of the fuzzy coalition $\mathbf{s} \in L(N)$. Similar to the crisp formulations we provide the following definitions for their fuzzy counterpart.

Definition 7. A real valued function $\mu: L(N) \times F G(N) \rightarrow \mathbb{R}$ is called f-additive if for $\mathbf{s} \in L(N)$ and any pair $v_{1}, v_{2} \in F G(N)$ such that $v_{1}+v_{2} \in F G(N)$, it holds that

$$
\mu\left(\mathbf{s}, v_{1}+v_{2}\right)=\mu\left(\mathbf{s}, v_{1}\right)+\mu\left(\mathbf{s}, v_{2}\right) .
$$

Definition 8. A real valued function $\mu: L(N) \times F G(N) \rightarrow \mathbb{R}$ is called f-linear on the class $F G(N)$ of games if it is f-additive and if for any $v$ on $F G(N)$ and $\mathbf{s} \in L(N)$ it holds that $\mu(\mathbf{s}, \alpha v)=\alpha \mu(\mathbf{s}, v)$ for any real number $\alpha$ such that $\alpha v \in F G(N)$.

Definition 9. A real valued function $\mu: L(N) \times F G(N) \rightarrow \mathbb{R}$ is called positive if $\mu(\mathbf{s}, v) \geq 0 \forall v \in F G(N), \mathbf{s} \in L(N)$.

Definition 10. Given $v \in F G(N)$, $\mathbf{s} \in L(N)$, based on $\mathbf{s}$, a fuzzy coalition $\mathbf{s}^{\prime} \in \mathbf{s}^{f}$ is called a carrier of $\mathbf{s}$ in $v$ if for any $\mathbf{t} \in \mathbf{s}^{f}, v(t)=v(t \wedge \mathbf{s})$.

The set of carriers of $\mathbf{s}$ in $v$ is denoted by $S_{C}(v, \mathbf{s})$. Let $i \in N$, and $\mathbf{s} \in L(N)$. Take the fuzzy coalition $\mathbf{s}^{i}=\left(s_{1}^{i}, s_{2}^{i}, \ldots, s_{i}^{i}, \ldots, s_{n}^{i}\right)$ satisfying $s_{j}^{i}=s_{i}$ when $i=j$ and $s_{j}^{i}=0$ otherwise.

Definition 11. Given a function $\mu: L(N) \times F G(N) \rightarrow \mathbb{R}$, a $\mu$-Share function on $F G(N)$ is a function $\Psi^{\mu}: F G(N) \rightarrow\left(\mathbb{R}_{+}^{n}\right)^{L(N)}$ that satisfies the following axioms, i.e., Axiom $\mathrm{FS}_{1}-\mathrm{FS}_{3}$ along with Axiom $\mathrm{FS}_{4}$ or Axiom $\mathrm{FS}_{5}$.

Axiom $\mathbf{F S}_{1}$ (f-Efficiency): For $\mathbf{s} \in L(N)$ we have, $\sum_{i \in N} \Psi_{i}^{\mu}(\mathbf{s}, v)=1$, and $\Psi_{i}^{\mu}(\mathbf{s}, v)=0$, for each $i \notin \operatorname{Supp}(\mathbf{s})$.

Axiom $\mathbf{F S}_{\mathbf{2}}$ (f-Carrier): If $\mathbf{s}^{\prime} \in \mathbf{s}^{f}$ is a fuzzy carrier for $\mathbf{s}$ in $v$, then

$$
\Psi_{i}^{\mu}\left(\mathbf{s}^{\prime}, v\right)=\Psi_{i}^{\mu}(\mathbf{s}, v), \quad \text { for all } \quad i \in N .
$$

Axiom $\mathbf{F S}_{\mathbf{3}}$ (f-Symmetry): If for $i, j \in N$ and $\mathbf{t} \in s^{f}$ with $t_{i}=t_{j}=0$, we have $v\left(\mathbf{t} \vee \mathbf{s}^{i}\right)=v\left(\mathbf{t} \vee \mathbf{s}^{j}\right)$, then

$$
\Psi_{i}^{\mu}(\mathbf{s}, v)=\Psi_{j}^{\mu}(\mathbf{s}, v) .
$$

Axiom $\mathbf{F S}_{4}\left(f \mu\right.$-additivity): For any pair $v_{1}, v_{2} \in F G(N)$ such that $v_{1}+v_{2} \in$ $F G(N)$, it holds that

$\mu\left(\mathbf{s}, v_{1}+v_{2}\right) \Psi_{i}^{\mu}\left(\mathbf{s}, v_{1}+v_{2}\right)=\mu\left(\mathbf{s}, v_{1}\right) \Psi_{i}^{\mu}\left(\mathbf{s}, v_{1}\right)+\mu\left(\mathbf{s}, v_{2}\right) \Psi_{i}^{\mu}\left(\mathbf{s}, v_{2}\right), \quad$ for all $\quad i \in N$. 
Axiom $\mathbf{F S}_{\mathbf{5}}\left(f \mu\right.$-linearity): For any pair $v_{1}, v_{2} \in F G(N)$ such that $v_{1}+v_{2} \in$ $F G(N)$, it holds that $\mu\left(\mathbf{s}, a v_{1}+b v_{2}\right) \Psi_{i}\left(\mathbf{s}, a v_{1}+b v_{2}\right)=a \mu\left(\mathbf{s}, v_{1}\right) \Psi_{i}^{\mu}\left(\mathbf{s}, v_{1}\right)+$ $b \mu\left(\mathbf{s}, v_{2}\right) \Psi_{i}^{\mu}\left(\mathbf{s}, v_{2}\right)$, for any pair of real numbers $a$ and $b$ such that $a v_{1}+b v_{2} \in$ $F G(N)$ for all $i \in N$.

It follows from the above definition that for any $v \in F G(N)$ and the function $\mu$, a Share function $\Psi^{\mu}$ gives a payoff $\Psi_{i}^{\mu}(\mathbf{s}, v)$ to player $i$ when she is involved in the fuzzy coalition $\mathbf{s}$ and satisfies the aforementioned axioms. In the following we define a Share function for a specific class of fuzzy games, namely the games in proportional value form and Choquet integral introduced by [10].

\section{Share functions on two special classes of fuzzy games}

We discuss two particular classes of fuzzy games namely, fuzzy games in proportional value form and in Choquet integral form, respectively, and obtain their corresponding Share functions.

\subsection{Share functions for fuzzy games in proportional value form}

To discuss the existence and uniqueness of the Share function for fuzzy games in proportional value form we have to use some classical results from [12. Recall that given a coalition $T \in 2^{N}$, the unanimity game $w_{T}$ is defined as follows. $w_{T}(R)=1$ if $T \subseteq R$ and $w_{T}(R)=0$ otherwise. For any $T \in 2^{N}, v \in G_{0}(N)$ can be expressed as

$$
v=\left(\sum_{T \in 2^{N}} c_{T}(v) w_{T}\right),
$$

where

$$
c_{T}(v)=\sum_{R \subseteq T}(-1)^{|T|-|R|} v(R) .
$$

TheOrem 8. For any $v \in F G_{p}(N)$ we have

$$
v=\left(\sum_{T \in 2^{N}} c_{T}(v) u_{T}\right),
$$

where for any $T \in 2^{N}, c_{T}(v)$ are given above and $u_{T}$ is given by

$$
u_{T}(\mathbf{s})=\sum_{p \in[0,1]} w_{S}\left(\mathbf{s}^{p}\right) \cdot p \quad \text { for all } \quad \mathbf{s} \in L(N) .
$$




\section{A SIMPLIFIED EXPRESSION OF SHARE FUNCTIONS FOR COOPERATIVE GAMES}

So with the help of above theorem, $v(\mathbf{s})$ can be re-written as follows:

$$
v(\mathbf{s})=\left(\sum_{\substack{T \in 2^{N} \\ c_{T}(v) \geq 0}} c_{T}(v) u_{T}(\mathbf{s})\right)-\left(\sum_{\substack{T \in 2^{N} \\ c_{T}(v)<0}}\left(-c_{T}(v)\right) u_{T}(\mathbf{s})\right) .
$$

Following theorem extends a similar theorem in [16] to the class $F G_{p}(N)$.

Theorem 9. Let $\mu: F G_{p}(N) \rightarrow \mathbb{R}$ be a real valued function. There exists a unique $\mu$-Share function $\Psi^{\mu}: F G_{p}(N) \rightarrow\left(\mathbb{R}_{+}^{n}\right)^{L(N)}$ that satisfies the axioms of $f$-efficiency $\left(F S_{1}\right)$, f-carrier $\left(F S_{2}\right)$, f-symmetry $\left(F S_{3}\right)$ and $f \mu$-additivity $\left(F S_{4}\right)$ if and only if $\mu$ is f-additive on $F G_{p}(N)$.

Proof. The proof proceeds in the line of [16. First we suppose $\Psi^{\mu}$ satisfies f-efficiency and $f \mu$-additivity. It follows that $\Psi^{\mu}$ is $\mu$-additive on $G_{0}(N)$. Thus we have

$$
\begin{aligned}
& \mu(\mathbf{s}, u+v) \sum_{i \in N} \Psi_{i}^{\mu}(\mathbf{s}, u+v) \\
& =\mu(\mathbf{s}, u+v)\left[\Psi_{1}^{\mu}(\mathbf{s}, u+v)+\cdots+\Psi_{|\operatorname{Supp}(\mathbf{s})|}^{\mu}(\mathbf{s}, u+v)\right] \\
& =\mu(\mathbf{s}, u) \sum_{i \in N} \Psi_{i}(\mathbf{s}, u)+\mu(\mathbf{s}, v) \sum_{i \in N} \Psi_{i}(\mathbf{s}, v)
\end{aligned}
$$

for any $u, v \in F G_{p}(N)$ such that $u+v \in F G_{p}(N)$. f-efficiency then implies that $\mu(\mathbf{s}, u+v)=\mu(\mathbf{s}, u)+\mu(\mathbf{s}, v)$. Hence $\mu$ is f-additive.

Secondly, we will show that we can have at most one Share function $\Psi^{\mu}$ : $F G_{p}(N) \rightarrow\left(\mathbb{R}_{+}^{n}\right)^{L(N)}$ satisfying the four axioms. Let $\Psi^{\mu}: F G_{p}(N) \rightarrow\left(\mathbb{R}_{+}^{n}\right)^{L(N)}$ be a function satisfying the four axioms. For a positively scaled unanimity game $\alpha u_{T} \in F G_{p}(N), \alpha>0$ and consequently a fuzzy carrier $\mathbf{s}^{\prime} \in \mathbf{s}^{f}$ for $\alpha u_{T} \in$ $F G_{p}(N)$, we obtain,

(i) $\Psi_{i}^{\mu}\left(\mathbf{s}, \alpha u_{T}\right)=\frac{1}{\left|\mathbf{s}^{\prime}\right|}$ when $\mathbf{s}_{i}^{\prime} \neq 0$.

(ii) $\Psi_{i}^{\mu}\left(\mathbf{s}, \alpha u_{T}\right)=0$ when $\mathbf{s}_{i}^{\prime}=0$.

Again for $\alpha u_{T}, \alpha>0$ from (i) and (ii) clearly $\Psi^{\mu}$ satisfies all the four axioms. Thus it follows that for any $\alpha u_{T}, \alpha>0$ the function $\Psi^{\mu}$ given by (i) and (ii) is the Share function satisfying the axioms of f-efficiency, f-carrier and f-symmetry if and only if $\mu$ is f-additive.

The uniqueness of $\Psi^{\mu}(\mathbf{s}, v)$ follows immediately. We next show that $\Psi^{\mu}(\mathbf{s}, v)$ satisfies the four axioms for an arbitrary $v$. The assumption of f-additivity of $\mu$ ensures f-efficiency as in the case of crisp games. Consequently, the f-carrier axiom also follows. Third, for any $\mathbf{t} \in L(N)$ with $t_{i}=0, t_{j}=0, v\left(\mathbf{t} \vee \mathbf{s}^{i}\right)=$ $v\left(\mathbf{t} \vee \mathbf{s}^{j}\right)$, then $c_{\mathbf{s}^{i}}(v)=c_{\mathbf{s}^{j}}(v)$, whereas for any other $\mathbf{t} \in s^{f}$ with non-zero 


\section{RAJIB BISWAKARMA — SURAJIT BORKOTOKEY — RADKO MESIAR}

weight $c_{T}(v), i$ and $j$ either both have nonzero memberships in $\mathbf{s}$ or both have zero memberships in $\mathbf{s}$. Hence it follows that $\Psi_{i}^{\mu}(\mathbf{s}, v)=\Psi_{j}^{\mu}(\mathbf{s}, v)=0$ when $s_{i}=0, s_{j}=0$.

Next for $s_{i} \neq 0, s_{j} \neq 0$,

$$
\begin{aligned}
& \Psi_{i}^{\mu}(\mathbf{s}, v)=\frac{1}{\mu(\mathbf{s}, v)}\left[\sum_{T \in C^{+}} \mu\left(\mathbf{s}, c_{T}(v) u_{T}\right) \frac{1}{|\operatorname{Supp}(\mathbf{s})|}-\sum_{T \in C^{-}} \mu\left(\mathbf{s},-c_{T}(v) u_{T}\right) \frac{1}{|\operatorname{Supp}(\mathbf{s})|}\right], \\
& \Psi_{j}^{\mu}(\mathbf{s}, v)=\frac{1}{\mu(\mathbf{s}, v)}\left[\sum_{T \in C^{+}} \mu\left(\mathbf{s}, c_{T}(v) u_{T}\right) \frac{1}{|\operatorname{Supp}(\mathbf{s})|}-\sum_{T \in C^{-}} \mu\left(\mathbf{s},-c_{T}(v) u_{T}\right) \frac{1}{|\operatorname{Supp}(\mathbf{s})|}\right] .
\end{aligned}
$$

So $\Psi^{\mu}$ satisfies the symmetry $\left(\mathrm{FS}_{3}\right)$ axiom. Finally, for any two games $u, v \in$ $F G_{p}(N)$ we have that $(u+v)(\mathbf{t})=\left(\sum_{T \in 2^{N}}\left(c_{T}(u)+c_{T}(v)\right) u_{T}\right)(\mathbf{t})$. Following f-additivity of $\mu$ this implies $\mu(\mathbf{s}, u+v) \Psi^{\mu}(\mathbf{s}, u+v)=\mu(\mathbf{s}, u) \Psi^{\mu}(\mathbf{s}, u)+\mu(\mathbf{s}, v) \Psi(\mathbf{s}, v)$ and hence $\Psi^{\mu}$ is $f \mu$-additive.

Theorem 10. For given positive numbers $\omega_{k}, k=1,2, \ldots, n$ and $i \in \mathbf{s}^{p}, p \in(0,1]$, let the function $\mu^{\omega}$ be defined by

$$
\mu^{\omega}(\mathbf{s}, v)=\sum_{i \in N} \sum_{i \in T \subseteq \mathbf{s}^{p}} \omega_{k} M_{T}^{i}(\mathbf{s}, v)=W(v),
$$

where $M_{T}^{i}(\mathbf{s}, v)=\{v(T)-v(T \backslash i)\}$ and $k=|T|$. Then the Share function $\Psi_{\omega}^{\mu}$ defined by

$$
\Psi_{\omega_{i}}^{\mu}(s, v)=\frac{\sum_{i \in T \subseteq s^{p}} \omega_{k} M_{T}^{i}(\mathbf{s}, v)}{W(v)}
$$

is the unique Share function satisfying the axioms of $f$-efficiency $\left(\mathrm{FS}_{1}\right)$, f-carrier $\left(\mathrm{FS}_{2}\right)$, f-symmetry $\left(\mathrm{FS}_{3}\right)$ and $f \mu^{\omega}$-additivity $\left(\mathrm{FS}_{4}\right)$ in $F G_{p}(N)$ whenever $\mu^{\omega}$ is positive.

Pr o of. By definition, $\mu^{\omega}$ is f-additive. Hence the existence and uniqueness of the Share function follows from Theorem 9. We show that $\Psi_{\omega}^{\mu}$ satisfies the four axioms with respect to $\mu^{\omega}$ on the class $F G_{p}(N)$ of $\mu^{\omega}$-positive games. Next we show that $\Psi_{\mu}^{\omega}$ satisfies the above four axioms. The f-efficiency and f-null player axioms are direct consequences of their crisp counterparts. Now if $s_{i} \neq 0, s_{j} \neq 0$ and any $\mathbf{t} \in L(N)$ with $t_{i}=0, t_{j}=0, v\left(\mathbf{t} \vee \mathbf{s}^{i}\right)=v\left(\mathbf{t} \vee \mathbf{s}^{j}\right)$ then we have that $M_{T}^{i}(\mathbf{s}, v)=M_{T}^{j}(\mathbf{s}, v)$ for $T \subseteq \mathbf{s}^{p}, p \in(0,1]$. It follows that $M_{T \cup\{i\}}^{i}(\mathbf{s}, v)=$ $M_{T \cup\{j\}}^{j}(\mathbf{s}, v)$ for all $T \subseteq \mathbf{s}^{p}, p \in(0,1]$ whenever $s_{i}=0, s_{j}=0$ and $M_{T}^{i}(\mathbf{s}, v)$ $=M_{T \cup\{j\} \backslash\{i\}}^{j}(\mathbf{s}, v)$ for all $T \subseteq \mathbf{s}^{p}, p \in(0,1]$ whenever $s_{i} \neq 0$ and $s_{j}=0$. Following the fact that $\omega_{k}$ depends only on the size of $T$, the symmetry axiom holds. Finally we have $\mu^{\omega}(\mathbf{s}, v) \Psi_{\omega_{i}}^{\mu}(\mathbf{s}, v)=\sum_{i \in T \subseteq \mathbf{s}^{p}} \omega_{k} M_{T}^{i}(\mathbf{s}, v)$. Since for all $i$ and $T$, it holds that $M_{T}^{i}(\mathbf{s}, a u+b v)=a M_{T}^{i}(\mathbf{s}, u)+b M_{T}^{i}(\mathbf{s}, v)$ it follows that $\Psi_{\omega}^{\mu}$ is $\mu^{\omega}$-additive. 


\section{A SIMPLIFIED EXPRESSION OF SHARE FUNCTIONS FOR COOPERATIVE GAMES}

\subsection{Examples of Share functions on fuzzy games in proportional value form}

In this section, we give the Shapley and Banzhaf Share functions for fuzzy games in proportional value form.

Definition 12. The Shapley Share function $\Psi^{\mu_{S}}$ on the class $F G(N)$ is defined by

$$
\Psi_{i}^{\mu_{S}}(\mathbf{s}, v)=\frac{\Phi_{i}^{S h}(\mathbf{s}, v)}{W(v)}, \quad \text { for all } \quad i \in N .
$$

Definition 13. The Banzhaf Share function $\Psi^{\mu_{B}}$ on the class $F G(N)$ defined by

$$
\Psi_{i}^{\mu_{B}}(\mathbf{s}, v)=\frac{\Phi_{i}^{B}(\mathbf{s}, v)}{W(v)}, \quad \text { for all } \quad i \in N .
$$

Theorem 11. Let the function $\mu_{S}$ be defined by $\mu_{S}(\mathbf{s}, v)=v(\mathbf{s})=W(v)$. Then the Shapley Share function $\Psi^{\mu_{S}}$ is the unique Share function satisfying the axioms of $f$-efficiency, f-carrier, f-symmetry and $f \mu_{S}$-linearity on $F G_{p}(N)$.

Proof. For $T \subseteq \mathbf{s}^{p}, p \in[0,1]$ with $|T|=k$, take $\omega_{k}=\frac{(k-1) !\left(\left|s^{p}\right|-k\right) !}{\left|s^{p}\right| !}$. Then from Theorem [10, we have $\mu^{\omega}$ as follows

$$
\mu^{\omega}(\mathbf{s}, v)=\sum_{i \in N} \sum_{i \in T \subseteq \mathbf{s}^{p}} \omega_{k} M_{T}^{i}(\mathbf{s}, v)=v(N)=\mu_{S}(\mathbf{s}, v) .
$$

Further, the Share function $\Psi_{\omega}^{\mu}$ as defined in Theorem 10 is given by

$$
\begin{aligned}
\Psi_{\omega i}^{\mu}(\mathbf{s}, v) & =\frac{\sum_{i \in T \subseteq \mathbf{s}^{p}} \omega_{k} M_{T}^{i}(\mathbf{s}, v)}{\mu^{\omega}(\mathbf{s}, v)} \\
& =\frac{\sum_{i \in T \subseteq \mathbf{s}^{p}} \frac{(k-1) !\left(\left|\mathbf{s}^{p}\right|-k\right) !}{\left|\mathbf{s}^{p}\right| !} M_{S}^{i}(\mathbf{s}, v)}{\mu_{S}(\mathbf{s}, v)} \\
& =\frac{\Phi_{i}^{S}(\mathbf{s}, v)}{v(\mathbf{s})} \\
& =\Psi_{i}^{S}(\mathbf{s}, v), \quad i \in N .
\end{aligned}
$$

This completes the proof.

Theorem 12. Let the function $\mu_{B}$ be defined by $\mu_{B}(s, v)=W(v)$. Then the Banzhaf Share function $\Psi^{\mu_{B}}$ is the unique Share function satisfying the axioms of f-efficiency, f-carrier, f-symmetry and $f \mu_{B}$-linearity on $F G_{p}(N)$. 
Proof. For $T \subseteq \mathbf{s}^{p}, p \in[0,1]$ with $|T|=k$, take $\omega_{k}=\frac{1}{2^{\left|s^{p}\right|-1}}$. Then, we have that $\mu^{\omega}$ as defined in Theorem 10 is given by

$$
\mu^{\omega}(\mathbf{s}, v)=\sum_{i \in N} \sum_{i \in T \subseteq \mathbf{s}^{p}} \omega_{k} M_{T}^{i}(\mathbf{s}, v)=\mu_{B}(\mathbf{s}, v)=W(v) .
$$

Further, the Share function $\Psi_{\omega}^{\mu}$ as defined in Theorem 10 is given by,

$$
\begin{aligned}
\Psi_{\omega i}^{\mu}(\mathbf{s}, v) & =\frac{\sum_{i \in T \subseteq \mathbf{s}^{p}} \omega_{k} M_{S}^{i}(\mathbf{s}, v)}{\mu^{\omega}(\mathbf{s}, v)} \\
& =\frac{\sum_{i \in T \subseteq \mathbf{s}^{p}} \frac{1}{2^{\left|\mathbf{s}^{p}\right|-1}} M_{T}^{i}(\mathbf{s}, v)}{\mu_{B}(\mathbf{s}, v)} \\
& =\frac{\Phi_{i}^{B}(\mathbf{s}, v)}{W(v)} \\
& =\Psi_{i}^{\mu_{B}}(\mathbf{s}, v), \quad i \in N .
\end{aligned}
$$

In the following example we obtain the numerical values of the Share functions for a fuzzy game in proportional value form.

Example 1. Let $N=\{1,2,3\}$ and $\mathbf{s}=(0.5,0.5,0.3) \in L(N)$. The crisp game $v \in G_{0}(N)$ is defined as follows. $v(1)=120, v(2)=150, v(3)=180$, $v(1,2)=450, v(1,3)=480, v(2,3)=480$ and $v(N)=840$. With $\mathbf{s}$ given as above, we obtain the worth of $\mathbf{s}$ under a fuzzy game in proportional value form namely $v(\mathbf{s})=279$. After some computations, the Shapley share is given by $\Psi^{\mu_{S}}(\mathbf{s}, v)=(0.37,0.43,0.20)$. In a similar way we get $W(v)=121.5$, consequently the Banzhaf share is found to be $\Psi^{\mu_{B}}(\mathbf{s}, v)=(0.25,0.31,0.44)$.

\subsection{Share functions on fuzzy games in the Choquet integral form}

Following similar procedure as in Theorem 10, we can have for $v \in F G_{C}(N)$,

$$
v=\left(\sum_{T \in 2^{N}} c_{T}(v) z_{T}\right)
$$

where

$$
c_{T}(v)=\sum_{R \subseteq T}(-1)^{|T|-|R|} v(R)
$$

and

$$
z_{T}(\mathbf{s})=\sum_{l=1}^{q(\mathbf{s})} w_{T}\left([\mathbf{s}]_{h_{l}}\right) \cdot\left(h_{l}-h_{l-1}\right) \quad \text { for all } \quad \mathbf{s} \in L(N)
$$




\section{A SIMPLIFIED EXPRESSION OF SHARE FUNCTIONS FOR COOPERATIVE GAMES}

It follows from the above discussion that $v(S)$ can be re-written as

$$
v(s)=\left(\sum_{\substack{T \in 2^{N} \\ c_{T}(v) \geq 0}} c_{T}(v) z_{T}(s)\right)-\left(\sum_{\substack{T \in 2^{N} \\ c_{T}(v)<0}}\left(-c_{T}(v)\right) z_{T}(s)\right) .
$$

Theorem 13. Let $\mu: F G_{C}(N) \rightarrow \mathbb{R}$ be a real valued function on the class $F G_{C}(N)$. There exists a unique $\mu$-Share function $\Psi^{\mu}: F G_{C}(N) \rightarrow\left(\mathbb{R}_{+}^{n}\right)^{L(N)}$ that satisfies the axioms of f-efficiency $\left(\mathrm{FS}_{1}\right)$, f-carrier $\left(\mathrm{FS}_{2}\right)$, f-symmetry $\left(\mathrm{FS}_{3}\right)$ and $f \mu$-additivity $\left(\mathrm{FS}_{4}\right)$ if and only if $\mu$ is f-additive on $F G_{C}(N)$.

Pr o of. The proof is similar to Theorem 9 and hence omitted.

Theorem 14. For given positive numbers $\omega_{k}$ with $k=1,2, \ldots, n$, let the function $\mu^{\omega}$ be defined by

$$
\mu^{\omega}(\mathbf{s}, v)=\sum_{i \in N} \sum_{l=1}^{q(\mathbf{s})}\left\{\sum_{i \in T \subseteq[\mathbf{s}]_{h_{l}}} \omega_{k} M_{T}^{i}(\mathbf{s}, v)\right\}\left(h_{l}-h_{l-1}\right)=W(v)
$$

where $M_{T}^{i}(\mathbf{s}, v)=\{v(T)-v(T \backslash i)\}$ and $k=|T|$. Then the Share function $\Psi_{\omega}^{\mu}$ defined by

$$
\Psi_{\omega i}^{\mu}(\mathbf{s}, v)=\frac{\sum_{l=1}^{q(\mathbf{s})}\left\{\sum_{i \in T \subseteq[\mathbf{s}]_{h_{l}}} \omega_{k} M_{T}^{i}(\mathbf{s}, v)\right\}\left(h_{l}-h_{l-1}\right)}{W(v)}
$$

is the unique Share function satisfying the axioms of $f$-efficiency $\left(\mathrm{FS}_{1}\right)$, f-carrier $\left(\mathrm{FS}_{2}\right)$, f-symmetry $\left(\mathrm{FS}_{3}\right)$ and $f \mu^{\omega}$-additivity $\left(\mathrm{FS}_{4}\right)$ on $F G_{C}(N)$ wherever $\mu^{\omega}$ is positive.

Pr o of. The proof is similar to the proof of Theorem 12 and hence omitted.

\subsection{Examples of Share functions on fuzzy games in Choquet integral form}

In this section, we discuss the Shapley and Banzhaf Share functions for fuzzy games in Choquet integral form.

Theorem 15. Let the function $\mu_{S}$ be defined by $\mu_{S}(s, v)=v(s)=W(v)$. Then the Shapley Share function $\Psi^{\mu_{S}}$ is the unique Share function satisfying the axioms of $f$-efficiency, $f$-carrier, f-symmetry and $f \mu_{S}$-linearity on $F G_{C}(N)$. 


\section{RAJIB BISWAKARMA — SURAJIT BORKOTOKEY — RADKO MESIAR}

Proof. For $T \subseteq[\mathbf{s}]_{h_{l}}$ with $|T|=k$, take $\omega_{k}=\frac{(k-1) !\left(\left|[\mathbf{s}]_{h_{l}}\right|-k\right) !}{\left|[s]_{h_{l}}\right| !}$. Then, we have that $\mu^{\omega}$ as defined in Theorem 11 given by

$$
\mu^{\omega}(\mathbf{s}, v)=\sum_{i \in N}\left\{\sum_{l=1}^{q(\mathbf{s})} \sum_{i \in T \subseteq[\mathbf{s}]_{h_{l}}} \omega_{k} M_{T}^{i}(\mathbf{s}, v)\right\}\left(h_{l}-h_{l-1}\right)=v(N)=\mu_{S}(\mathbf{s}, v) .
$$

Further, the Share function $\Psi_{\omega}^{\mu_{S}}$ as defined in Theorem 11 is given by

$$
\begin{aligned}
\Psi_{\omega i}^{\mu_{S}}(\mathbf{s}, v) & =\frac{\left\{\sum_{l=1}^{q(\mathbf{s})} \sum_{i \in T \subseteq[\mathbf{s}]_{h_{l}}} \omega_{k} M_{T}^{i}(\mathbf{s}, v)\right\}\left(h_{l}-h_{l-1}\right)}{\mu_{S}(\mathbf{s}, v)} \\
& =\frac{\sum_{l=1}^{q(\mathbf{s})}\left\{\sum_{i \in T \subseteq[\mathbf{s}]_{h_{l}}} \frac{(k-1) !\left(\left|[s]_{h_{l}}\right|-k\right) !}{\left|[\mathbf{s}]_{h_{l}}\right| !} M_{T}^{i}(\mathbf{s}, v)\right\}\left(h_{l}-h_{l-1}\right)}{\mu_{S}(\mathbf{s}, v)} \\
& =\frac{\Phi_{i}^{S h}(\mathbf{s}, v)}{v(\mathbf{s})}=\Psi_{i}^{\mu_{S}}(\mathbf{s}, v), \quad i \in N .
\end{aligned}
$$

Theorem 16. Let the function $\mu_{B}$ be defined by $\mu_{B}(\mathbf{s}, v)=W(v)$. Then the Banzhaf Share function $\Psi^{\mu_{B}}$ is the unique Share function satisfying the axioms of f-efficiency, f-carrier, f-symmetry and $f \mu_{B}$-linearity on $F G_{C}(N)$.

Pr o of. For $T \subseteq[\mathbf{s}]_{h_{l}}$ with $|T|=k$, take $\omega_{k}=\frac{1}{{ }_{2}{ }^{\left|[\mathbf{s}]_{h_{l}}\right|-1}}$. Then, we have that $\mu^{\omega}$ as defined in Theorem 11 is given by

$$
\mu^{\omega}(\mathbf{s}, v)=\sum_{i \in N} \sum_{l=1}^{q(\mathbf{s})}\left\{\sum_{i \in T \subseteq[\mathbf{s}]_{h_{l}}} \omega_{k} M_{T}^{i}(\mathbf{s}, v)\right\}\left(h_{l}-h_{l-1}\right)=\mu_{B}(\mathbf{s}, v)=W(v) .
$$

Further, the Share function $\Psi_{\omega}^{\mu_{B}}$ defined in Theorem 11 is given by

$$
\begin{aligned}
\Psi_{\omega i}^{\mu_{B}}(\mathbf{s}, v) & =\frac{\sum_{l=1}^{q(\mathbf{s})}\left\{\sum_{i \in T \subseteq[\mathbf{s}]_{h_{l}}} \omega_{k} M_{S}^{i}(\mathbf{s}, v)\right\}\left(h_{l}-h_{l-1}\right)}{\mu^{\omega}(\mathbf{s}, v)} \\
& =\frac{\sum_{l=1}^{q(\mathbf{s})}\left\{\sum_{i \in T \subseteq[\mathbf{s}]_{h_{l}}} \frac{1}{2^{\left|[\mathbf{s}]_{h_{l}}\right|-1}} M_{T}^{i}(\mathbf{s}, v)\right\}\left(h_{l}-h_{l-1}\right)}{\mu_{B}(\mathbf{s}, v)} \\
& =\frac{\Phi_{i}^{B}(\mathbf{s}, v)}{W(v)}=\Psi_{i}^{\mu_{B}}(\mathbf{s}, v), \quad i \in N .
\end{aligned}
$$

Following example computes the Shapley and Banzhaf Share values for a fuzzy game in Choquet integral form. 


\section{A SIMPLIFIED EXPRESSION OF SHARE FUNCTIONS FOR COOPERATIVE GAMES}

EXAmple 2. Let us keep $N=\{1,2,3\}$ and $\mathbf{s}=(0.5,0.5,0.3) \in L(N)$. The coalitional values of the associated crisp game $v \in G_{0}(N)$ are given as follows. $v(1)=120, v(2)=150, v(3)=180, v(1,2)=450, v(1,3)=480, v(2,3)=480$ and $v(N)=840$. When $v \in F G_{C}(N)$, i.e., fuzzy game in Choquet integral form we obtain $v(s)=342$. Consequently the Shapley share is given by $\Psi^{\mu_{S}}(\mathbf{s}, v)=$ $(0.35,0.38,0.27)$. Similarly, taking $W(v)=621.25$ the Banzhaf share is found to be $\Psi^{\mu_{B}}(\mathbf{s}, v)=(0.33,0.37,0.3)$.

\section{Conclusion}

We have discussed Share functions under fuzzy environment. The Shapley Share function and Banzhaf Share function are obtained for the class $F G_{p}(N)$ and $F G_{C}(N)$ of fuzzy games in proportional value form and Choquet integral form, respectively. The characterizing properties are studied. In the future, Share functions for other classes of fuzzy games will be investigated.

Acknowledgement. The authors acknowledge the comments and suggestions of the editor and the anonymous referee.

\section{REFERENCES}

[1] AUBIN, J. P.: Cooperative fuzzy games, Math. Oper. Res. 6 (1988), 1-13.

[2] AUBIN, J. P.: Mathematical Methods of Game and Economic Theory. (Reprint of the 1982 revised ed.), Dover Publ., Mineola, NY, 2007.

[3] ALVAREZ-MOZOS, M.-VAN DEN BRINK, R.-VAN DER LAAN, G.-TEJADA, O.: Share functions for cooperative games with levels structure of cooperation, European J. Oper. Res. 244 (2013), 167-179.

[4] AUMANN, R. J.-SHAPLEY, L. S.: Values of Non-Atomic Games. Princeton Univ. Press, Princeton, NJ, 1974.

[5] AUMANN, R.-HART, S. (EDS.): Handbook of Game Theory with Economic Applications, in: Handbooks in Economics, Vol. 11, Elsevier, Amsterdam, 2002.

[6] BANZHAF, J. F: Weighted voting doesn't work: a mathematical analysis, Rutgers Law Review 19 (1965), 317-343.

[7] BUTNARIU, D.: Stability and Shapley value for an n-persons fuzzy game, Fuzzy Sets Syst. 4 (1980), 63-72.

[8] CHOQUET, G.: Theory of capacities, Ann. Inst. Fourier (Grenoble) 5 (1953), 131-295.

[9] LI, S.-ZHANG, Q.: A simplified expression of the Shapley function for fuzzy game, European J. Oper. Res. 196 (2009), 234-245.

[10] MENG, F. Y.-ZHANG, Q.: The Shapley Value on a kind of cooperative fuzzy games, J. Comput. Inform. Syst. 7 (2011), 1846-1854.

[11] MENG, F. Y.-ZHANG, Q.: The Shapley function for fuzzy cooperative games with multilinear extension form, European J. Oper. Res. 23 (2010), 644-650.

[12] SHAPLEY, L. S.: A value for n-person games, Ann. of Math. Stud. 28 (1953), 307-317. 


\section{RAJIB BISWAKARMA — SURAJIT BORKOTOKEY — RADKO MESIAR}

[13] SPRUMONT, Y.: Population monotonic allocation schemes for cooperative games with transferable utility, Games Econom. Behav. 2 (1990), 378-394.

[14] TAN, C.-JIANG, Z. Z.-CHEN, X.-IP, W. H.: A Banzhaf function for fuzzy game, IEEE Trans. Fuzzy Syst. 22 (2014), 1489-1502.

[15] TSURUMI, M.-TANINO, T.-INUIGUCHI, M.: A Shapley function on a class of cooperative fuzzy games, Eur. J. Oper. Res. 129 (2001), 596-618.

[16] VAN DER LAAN, G.-VAN DEN BRINK, R.: Axiomatizations of a class of share functions on n-person games, Theory and Decision 44 (1998), 117-148.

[17] VAN DER LAAN, G.-VAN DEN BRINK, R.: The normalized Banzhaf value and the Banzhaf share function, Int. J. Math. Game Theory Algebra 9 (1999), 65-84.

[18] VAN DER LAAN, G.-VAN DEN BRINK, R.: A class of consistent share functions for games in coalition structure, Games Econom. Behav. 51 (2005), 193-212.

Received April 14, 2017
Rajib Biswakarma

Surajit Borkotokey

Department of Mathematics

Dibrugarh University

Dibrugarh

Assam

INDIA-786004

E-mail: rajib01101987@gmail.com surajitbor@yahoo.com

Radko Mesiar

Slovak University of Technology

Department of Mathematics

Radlinského 11

SK-810 05 Bratislava

SLOVAKIA

E-mail: mesiar@math.sk 